\title{
Viscosity and Thermal Relaxation for a resonantly interacting Fermi gas
}

\author{
G. M. Bruun \\ Niels Bohr Institute, Blegdamsvej 17, DK-2100 Copenhagen Ø, Denmark \\ H. Smith \\ Ørsted Laboratory, Niels Bohr Institute, Universitetsparken 5, DK-2100 Copenhagen Ø, Denmark.
}

(Dated: August 6, 2021)

\begin{abstract}
The viscous and thermal relaxation rates of an interacting fermion gas are calculated as functions of temperature and scattering length, using a many-body scattering matrix which incorporates medium effects due to Fermi blocking of intermediate states. These effects are demonstrated to be large close to the transition temperature $T_{c}$ to the superfluid state. For a homogeneous gas in the unitarity limit, the relaxation rates are increased by nearly an order of magnitude compared to their value obtained in the absence of medium effects due to the Cooper instability at $T_{c}$. For trapped gases the corresponding ratio is found to be about three due to the averaging over the inhomogeneous density distribution. The effect of superfluidity below $T_{c}$ is considered to leading order in the ratio between the energy gap and the transition temperature.
\end{abstract}

Pacs Numbers: 03.75.Ss, 05.30.Fk,51.20.+d

\section{INTRODUCTION}

The properties of resonantly interacting fermions have been under intense investigation during the last few years, stimulated by the ability to manipulate the interatomic interaction through the use of Feshbach resonances. In this way it has been possible to create molecular Bose-Einstein condensates from weakly bound molecules consisting of two fermionic atoms. The molecular condensates are formed when the scattering length is positive, corresponding to an effective repulsion between particles. If the interaction is attractive (the scattering length negative) the atoms may pair up in a manner similar to the way in which electrons form Cooper pairs in superconducting metals. The important dimensionless parameter for an interacting, degenerate fermion gas is the product of the Fermi wave number $k_{\mathrm{F}}$, given by $k_{\mathrm{F}}^{3}=3 \pi^{2} n$ where $n$ is the fermion density, and the scattering length $a$. For attractive interactions, and for $k_{\mathrm{F}}|a|$ much less than unity (which we refer to as the weak-coupling limit), the transition temperature $T_{c}$ to the superfluid phase is very much less than the Fermi temperature $T_{\mathrm{F}}$. Near Feshbach resonances it is possible to achieve values of $k_{\mathrm{F}}|a|$ that are much larger than unity (the strong-coupling limit), and in this case the expected transition temperature is comparable to the Fermi temperature, typically about one quarter of $T_{\mathrm{F}}$, depending on the model used for the effective scattering matrix.

In this work we demonstrate how many-body effects have a significant influence on the viscous and thermal relaxation rates at temperatures near the transition temperature to the superfluid state. The many-body effects are due to Fermi blocking of intermediate states in the effective scattering matrix. Such effects are known to influence equilibrium quantities such as chemical potential and total energy, but - as we shall see below - their influence on properties like thermal or viscous relaxation is much more pronounced. Thermal relaxation rates may be determined directly experimentally [1, 2], and viscous relaxation rates can be extracted from the attenuation of collective modes. Our work is an extension of [3] , in which the viscous relaxation rate was calculated using the vacuum scattering matrix, and we shall refer to this paper as I in the following.

\section{VISCOSITY AND THERMAL RELAXATION}

In this section we discuss the relation between the thermal and viscous relaxation rates for a two-component Fermi gas trapped in a harmonic-oscillator potential, with an equal number of particles in each component. The interaction between the fermions is taken to be attractive, $a<0$, and we consider fermions in their normal state, at $T>T_{c}$. For bosons in a harmonic trap, at temperatures above the Bose-Einstein transition temperature, the thermal and viscous relaxation rates have been shown to be simply related, differing only by an overall factor of two [4]. As one might expect, the same relation applies to the fermion gas, since the factor of two originates in the equality of kinetic and potential energies for a particle in a harmonic trap. We briefly indicate the line of reasoning which leads to this result and refer the reader to [4] for details.

We consider a gas of particles with mass $m$, trapped in an axially-symmetric harmonic-oscillator potential of the form

$$
V(\mathbf{r})=\frac{m}{2}\left(\omega_{\perp}^{2} r_{\perp}^{2}+\omega_{z}^{2} z^{2}\right),
$$

where $r_{\perp}^{2}=x^{2}+y^{2}$. For such a system we define the spatially averaged viscous relaxation rate by

$$
\frac{1}{\tau_{\eta}}=\frac{\int d^{3} r(X, H[X])}{\int d^{3} r(X, X)}
$$

where the symbols have the same meaning as in I. In particular $H$ is an integral operator derived from the col- 
lision integral of the Boltzmann equation, and $X$ is a trial function given by

$$
X=v_{y} p_{x}
$$

with $\mathbf{p}$ being the particle momentum and $\mathbf{v}$ the group velocity, equal to $\mathbf{p} / m$ in the normal state. In carrying out the spatial average we treat the trapping potential in the Thomas-Fermi approximation via a locally varying chemical potential $\mu(\mathbf{r})=\mu-V(\mathbf{r})$. The time $\tau_{\eta}$ determines the nature and the damping of the collective modes in the trapped atomic gases [3].

The measurement of thermal relaxation rates has proven a useful tool for investigating the properties of trapped gases 1, 2]. In such an experiment, the gas is "heated" preferentially in one spatial direction, and the relaxation toward equilibrium with a uniform temperature in all directions is determined from the time evolution of the rms cloud radii. The thermal relaxation rate can be extracted from the time-dependent aspect ratio. In analogy with the viscous relaxation rate, one may obtain a variational expression for the relaxation time of temperature anisotropies in a trap [4],

$$
\frac{1}{\tau_{T}}=\frac{\int d^{3} r\left(\Phi_{T}, H\left[\Phi_{T}\right]\right)}{\int d^{3} r\left(\Phi_{T}, \Phi_{T}\right)}
$$

where the trial function is

$$
\Phi_{T}=p_{z}^{2}-p^{2} / 3+\frac{m^{2}}{3}\left(2 \omega_{z}^{2} z^{2}-\omega_{\perp}^{2} r_{\perp}^{2}\right) .
$$

The momentum-dependent part of this trial function involves only a $l=2$ spherical harmonic in momentum space. If the spatial part of the trial function were neglected, the viscous and thermal relaxation rates would be identical, since the trial function (3) also involves only $l=2$ spherical harmonics. Since collisions relax only momentum anisotropies, the spatial part of (5) does not contribute to the numerator in (4), whereas it gives the same contribution to the denominator as the momentum part. It therefore follows from comparing (2) and (4) that the thermal and viscous relaxation rates are related by

$$
\tau_{T}=2 \tau_{\eta}
$$

The viscous relaxation rate is thus directly accessible experimentally via measurements of the thermal relaxation rate as in 1, 2].

\section{COLLISIONS}

In order to calculate the viscous relaxation rate, we need the particle-particle scattering cross section. The differential cross section $d \sigma / d \Omega$ for $s$ wave scattering is related to the scattering matrix $\mathcal{T}$ by

$$
\frac{d \sigma}{d \Omega}=\frac{m^{2}|\mathcal{T}|^{2}}{\left(4 \pi \hbar^{2}\right)^{2}}
$$

An often used approximation close to a resonance is to use the so-called unitarized vacuum scattering matrix

$$
\mathcal{T}_{\text {uni }}=\frac{4 \pi \hbar^{2} a}{m} \frac{1}{1+i q a}
$$

where $a$ is the scattering length and $\hbar q$ the relative momentum of the scattering particles. In the present paper, we shall extend the calculation carried out in I of the viscous relaxation rate to cover the temperature region near $T_{c}$ where medium effects absent in the approximation (8) turn out to be important.

Close to a Feshbach resonance, the scattering length can be written in the phenomenological form

$$
a=a_{\mathrm{bg}}\left(1-\frac{\Delta B}{B-B_{0}}\right) .
$$

Here, $\Delta B$ is the width of the resonance, $a_{\mathrm{bg}}$ the background scattering length coming from non-resonant scattering processes, and $B_{0}$ the position of the resonance. For atomic gases close to a Feshbach resonance, a multichannel effective theory for atom-atom scattering was recently presented [5]. The scattering matrix including medium effects was shown to be given by

$$
\mathcal{T}(\omega, \mathbf{K})=\frac{\mathcal{T}_{\mathrm{bg}}}{\left(1+\frac{\Delta \mu \Delta B}{\hbar \tilde{\omega}+h(\omega, \mathbf{K})-\Delta \mu\left(B-B_{0}\right)}\right)^{-1}-\mathcal{T}_{\mathrm{bg}} \Pi(\omega, \mathbf{K})},
$$

where $\hbar \mathbf{K}$ is the center-of-mass momentum of the scattering particles and $\omega$ the frequency. Here $\tilde{\omega}=\omega-\hbar K^{2} / 4 m$ and $\mathcal{T}_{\mathrm{bg}}=4 \pi a_{\mathrm{bg}} \hbar^{2} / m$, while $\Delta \mu$ is the magnetic moment of the Feshbach molecule. The pair propagator $\Pi$ is

$$
\begin{aligned}
\Pi(\omega, \mathbf{K})=\int \frac{d^{3} q^{\prime}}{(2 \pi)^{3}} & {\left[\frac{1-f^{0}\left(\frac{1}{2} \mathbf{K}-\mathbf{q}^{\prime}\right)-f^{0}\left(\frac{1}{2} \mathbf{K}+\mathbf{q}^{\prime}\right)}{\hbar \omega+i \delta-\frac{\hbar^{2} K^{2}}{4 m}-\frac{\hbar^{2} q^{\prime 2}}{m}}\right.} \\
+ & \left.\frac{1}{\hbar^{2} q^{\prime 2} / m-i \delta}\right]
\end{aligned}
$$

where $f^{0}$ is the equilibrium Fermi function. The quantity $h(\omega, \mathbf{K})$ in (10) describes effects coming from the fact that the Feshbach state is a composite two-fermion object, but it can be neglected for most resonances of experimental interest [5] and we therefore set it equal to zero, $h(\omega, \mathbf{K})=0$. For the calculation of transport coefficients such as the viscosity, the scattering matrix has to be evaluated on-shell with $\hbar \omega$ equal to the energy of the two scattering particles, i.e. $\hbar \omega=\hbar^{2} K^{2} / 4 m+\hbar^{2} q^{2} / m$. In a vacuum, the on-shell version of (10) with $h(\omega, \mathbf{K})=0$ reduces to

$$
\mathcal{T}=\frac{\frac{4 \pi \hbar^{2}}{m} a_{\mathrm{bg}}}{\left(1+\frac{\Delta \mu \Delta B}{\hbar^{2} q^{2} / m-\Delta \mu\left(B-B_{0}\right)}\right)^{-1}+i a_{\mathrm{bg}} q}
$$

We shall use (10) for calculating the low-temperature viscosity for a resonantly interacting gas. 
The approximation (8) for the scattering matrix neglects two effects as compared to the many-body expression (10). Firstly, even the vacuum limit (12) of (10) is not identical to (8); they agree only when the $\hbar^{2} q^{2} / m$ term in (12) can be neglected. This term corresponds to an effective range of the atom-atom interaction. Here we consider only broad resonances such as the one at $B \simeq 201.6 \mathrm{G}$ for ${ }^{40} \mathrm{~K}$ or the one at $B \simeq 830 \mathrm{G}$ for ${ }^{6} \mathrm{Li}$, for which the effective range can be safely neglected [5, $[$ ]

Secondly, the approximation given by (8) neglects medium effects, which are included in (10) via the propagator (11). These effects come from the occupation of open-channel states. One effect of the medium is to shift the resonance position away from its vacuum value $B_{0}[\underline{5}]$. More importantly for the present purpose, medium effects cause a significant increase in the scattering rate for $T \rightarrow 0$ [7] due to the Fermi blocking of the pair states into which the molecular state can decay. The decay of the molecular state in a vacuum is the origin of the iqa terms in the denominator of (8) and (12). These terms are replaced by $\mathcal{T}_{\mathrm{bg}} \Pi$ in (10) when medium effects are taken into account. At low temperatures, this term is significantly reduced from the vacuum value due to the Fermi-blocking factors in (11); the lifetime of the resonant state is increased leading to a corresponding increase in the scattering rate. We shall demonstrate that this effect is significant close to the superfluid transition temperature $T_{c}$.

\section{HOMOGENEOUS SYSTEM}

We now present results for the viscosity of a homogeneous system. The viscosity is calculated as a function of temperature for a constant density $n$. We calculate the thermodynamic potential $\Omega$ for fixed $n$ within the ladder approximation as a function of temperature, taking into account two-body scattering processes with a scattering matrix given by (10). The chemical potential $\mu$ is determined by the condition $-\partial_{\mu} \Omega=n$. Our calculation of $\Omega$ is identical to the one described in Ref. [6] where more details are given. Once the chemical potential is determined, we calculate $\eta$ using Eq. (16) of I with the cross section given by (7) and (10). The calculation of the chemical potential and the viscosity requires multi-dimensional integrations which are performed numerically using two different methods to check the accuracy. The first method uses convoluted 1D integration routines and the other is based on multidimensional Monte-Carlo integration. Both yield the same result for the chemical potential and the viscosity within less than $1 \%$, thus confirming the accuracy of the numerical calculations.

\section{A. Low and high temperature limits}

Before we present any results, let us first discuss what can be calculated analytically. For low temperatures $T / T_{\mathrm{F}} \ll 1\left(k T_{\mathrm{F}}=\hbar^{2} k_{\mathrm{F}}^{2} / 2 m\right)$, the Fermi factors in the collision integral (see Eq. (13) in I) restrict the available phase for the scattering particles such that their momenta are close to the Fermi surface. This simplifies the integrations and assuming the simple unitarized vacuum form (8) for the scattering matrix $\left(\mathcal{T}=\mathcal{T}_{\text {uni }}\right)$, an analytical result for the viscosity can be obtained using standard Fermi liquid tricks [8]. A lengthy but straightforward analysis yields for $k_{\mathrm{F}}|a| \ll 1$ and $T \rightarrow 0$

$$
\eta=\frac{3 n \hbar}{8 \pi\left(k_{\mathrm{F}} a\right)^{2}} \frac{\epsilon_{\mathrm{F}}^{2}}{(k T)^{2}}
$$

with $\epsilon_{\mathrm{F}}=\hbar^{2} k_{\mathrm{F}}^{2} / 2 m$. Equation (13) is based on $T \ll T_{\mathrm{F}}$ and the assumption $\mathcal{T}=\mathcal{T}_{\text {uni }}$. This assumption works well for weak coupling with $T_{c} \ll T_{\mathrm{F}}$ : there is then a temperature regime for which $T \ll T_{\mathrm{F}}$ such that all scattering momenta are confined to the Fermi surface, and $T \gg T_{c}$ such that medium effects can be neglected. However, for strong coupling $T_{c}$ becomes comparable to $T_{\mathrm{F}}$ and there is no temperature regime for which $T \ll T_{\mathrm{F}}$ and $T \gg T_{c}$.

In the classical limit $T \gg T_{\mathrm{F}}$, an analytical expression for the viscosity can also be obtained using $\mathcal{T}=\mathcal{T}_{\text {uni }}$ :

$$
\begin{gathered}
\eta=\frac{5 \sqrt{\pi m k T}}{8 \bar{\sigma}} \\
=\frac{5 \sqrt{m k T}}{32 \sqrt{\pi}} \times \begin{cases}a^{-2} & , T \ll T_{a} \\
3 m k T / \hbar^{2} & , T \gg T_{a}\end{cases}
\end{gathered}
$$

with $k T_{a}=\hbar^{2} /\left(m a^{2}\right)$ and

$$
\bar{\sigma}=\frac{4 \pi a^{2}}{3} \int_{0}^{\infty} d x x^{7} e^{-x^{2}}\left(1+x^{2} T / T_{a}\right)^{-1}
$$

the momentum averaged cross section. As opposed to the low-temperature limit, the assumption $\mathcal{T}=\mathcal{T}_{\text {uni }}$ is reasonable for $T \gg T_{\mathrm{F}}$, even in the strong-coupling case, since medium effect on the scattering matrix are suppressed at high temperatures.

\section{B. Numerical results}

Fig. 1 1 shows the viscosity $\eta$ in the weak-coupling limit $k_{\mathrm{F}}|a| \ll 1$. We have chosen parameters in (10) corresponding to a generic weak interaction characterized by background scattering with $k_{\mathrm{F}} a_{\mathrm{bg}}=-0.1, \Delta B /(B-$ $\left.B_{0}\right)=0.0012$, and a negligibly small effective range. For weak interaction, the scattering matrix reduces to $4 \pi \hbar^{2} a_{\mathrm{bg}} / m$ and there are no effects of the medium as long as $T$ is well above $T_{c} \ll T_{\mathrm{F}}$. In fact, the solid line in Fig. 1 is indistinguishable from the result obtained if we had used $4 \pi \hbar^{2} a_{\mathrm{bg}} / m$ for the scattering matrix of (10) 


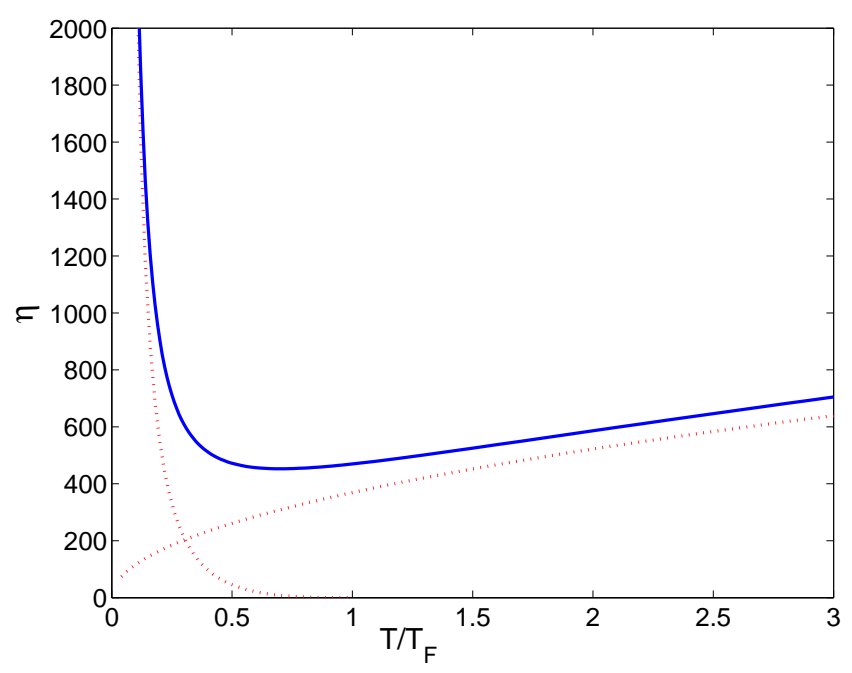

FIG. 1: (Color online) The viscosity $\eta$ in units of $n \hbar$ for background scattering. The solid line is the numerical result with the scattering matrix (10), the dotted lines are the low and high temperature limits.

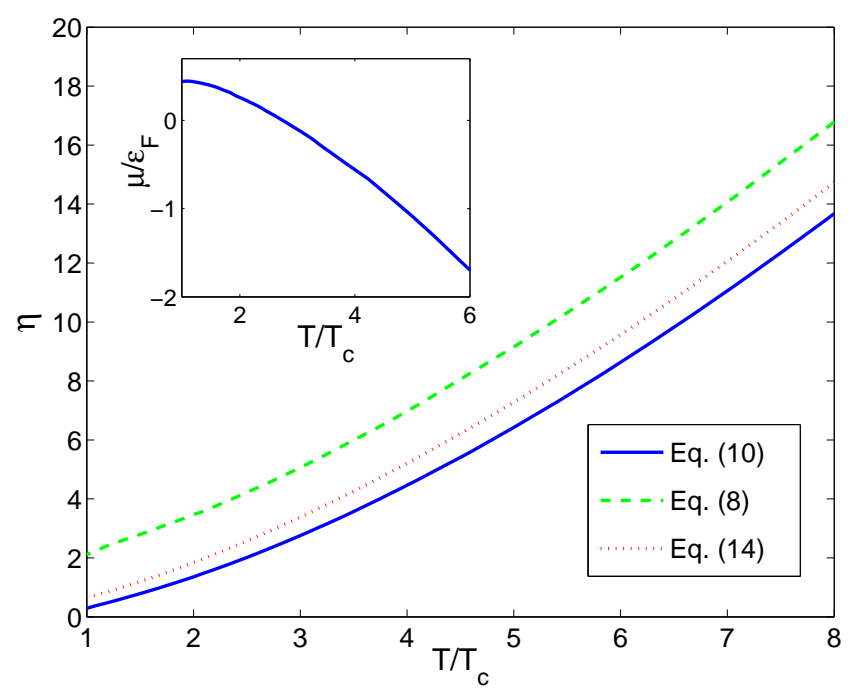

FIG. 2: (Color online) The viscosity $\eta$ in units of $n \hbar$ for resonant scattering as a function of temperature for $T \geq T_{c}$. The solid line is the numerical result with the full scattering matrix (10), the dashed line the numerical result using the vacuum approximation (8). The dotted line is the low-temperature result (14). The inset shows the chemical potential.

as expected. We see that the viscosity approaches the low- and high-temperature forms given by (13) and (14) as expected, with a minimum located at $T \approx 0.7 T_{\mathrm{F}}$.

Fig. 2 shows the viscosity $\eta$ in the strong-coupling limit. We have chosen parameters in (10) corresponding to a generic resonant interaction characterized by $k_{\mathrm{F}} a=-11.8$ and a negligible effective range. For this set of parameters very close to resonance, we find the critical temperature for the superfluid transition to be $T_{c} \approx 0.26 T_{\mathrm{F}}$ in excellent agreement with earlier numer- ical BEC-BCS crossover results based on a similar approximation for the thermodynamic potential 9,10 .

Just above the critical temperature $T_{c}$, the viscosity is reduced almost by an order of magnitude (a factor $\approx 7.5$ ) as compared to the value obtained using the vacuum scattering matrix. The reduction reflects the increase in the scattering rate due to Fermi blocking of the molecule decay as explained in Sec. III These medium effects cause the zero center-of-mass $(\mathrm{COM})$ momentum $\mathcal{T}$ matrix to diverge at $T_{c}$, which is the signature of the Cooper instability. Thus, we expect the increase in the scattering rate due to the medium to be significant for temperatures close to $T_{c}$, regardless of the value of the coupling strength. This is confirmed by numerical calculations for different values of the coupling: Medium effects on the scattering rate and thus the viscosity are significant when

$$
\frac{T-T_{c}}{T_{c}} \lesssim 1
$$

In the high temperature limit on the other hand, the medium effects on the scattering are suppressed as expected and the viscosity approaches its classical value as can be seen from Fig. 2 We conclude that the medium increases the scattering rate significantly as compared to the vacuum value for temperatures close to $T_{c}$ due to the Cooper instability.

Note that since $k T_{c} / \mu\left(T_{c}\right) \approx 0.6$ is rather large for strong coupling, the $T^{-2}$ increase in the viscosity coming from the phase space blocking in the collision integral is not observed for $T \geq T_{c}$ in Fig. 2

The above results show that the minimum viscosity for a Fermi gas in the normal phase for $k_{\mathrm{F}}|a| \rightarrow \infty$ and $T=T_{c}$ is

$$
\eta_{\min }=\alpha n \hbar
$$

with $\alpha \approx 0.15$. This minimum is significantly less than the value $\alpha \approx 1.1$ obtained when the vacuum scattering matrix given by (8) is used. We caution that the minimum value of $\eta / n \hbar$ obtained in (17) depends on the validity of our starting point, the semiclassical kinetic equation. A simple relaxation-time approximation to the collision integral, yielding the low-temperature viscosity $\eta \approx m n v_{\mathrm{F}}^{2} \tau / 5$, would result in $\eta \approx 0.4 n \hbar$ if $\tau=\hbar / \epsilon_{\mathrm{F}}$. However, for such small values of $\tau$ the semiclassical kinetic equation may need modification due to the spectral broadening of the momentum states. It is interesting to note that a related problem, the viscosity of a hot quarkgluon plasma, was recently analyzed within the Kubo formalism [11] which yielded results that differed significantly from those obtained from the Boltzmann equation.

\section{Effects of superfluidity}

The viscosity of a superfluid gas of fermions has been investigated extensively in the context of superfluid 
${ }^{3} \mathrm{He}$ [12. Below the transition temperature $T_{c}$, the viscosity drops rapidly as a function of temperature, the relative decrease in viscosity being of order $\Delta / k T_{c}$. The physical reason for the large drop is the change in the quasiparticle dispersion relation and the modification of the collision operator. For the purpose of illustration we shall here discuss the two effects separately, although they must of course be treated together in a consistent calculation.

Firstly, the group velocity is changed in the superfluid state, since the dispersion relation of Bogoliubov quasiparticles is

$$
E=\sqrt{\xi^{2}+\Delta^{2}}
$$

where $\xi=p^{2} / 2 m-\mu$ and $\Delta$ is the energy gap. The group velocity $\mathbf{v}_{g}=\partial E / \partial \mathbf{p}$ in the superfluid state is thus

$$
\mathbf{v}=\hat{\mathbf{p}} v_{\mathrm{F}} \frac{\xi}{E},
$$

where $\hat{\mathbf{p}}$ is a unit vector along $\mathbf{p}$ and $v_{\mathrm{F}}=\hbar k_{\mathrm{F}} / m$ is the Fermi velocity. This affects the velocity component $v_{y}$ appearing on the left hand side of the kinetic equation and in the expression for the momentum current density. To illustrate the effect of the change in group velocity, let us make a relaxation time approximation to the collision operator occurring in the Boltzmann equation. The shear viscosity is obtained by assuming a flow velocity $\mathbf{u}$ of the form $\mathbf{u}=\left(u_{x}(y), 0,0\right)$ and linearizing in the gradient $\partial u_{x} / \partial y$, as a result of which the kinetic equation for the non-equilibrium distribution function $f$ becomes

$$
-\frac{\partial u_{x}}{\partial y} v_{y} p_{x} \frac{\partial f^{0}}{\partial \epsilon}=-\frac{f-f^{0}}{\tau} .
$$

The shear viscosity $\eta$ relates the momentum current density $\Pi_{x y}$, given by

$$
\Pi_{x y}=2 \int \frac{d \mathbf{p}}{(2 \pi \hbar)^{3}} v_{y} p_{x} f,
$$

to the gradient of the flow velocity according to $\Pi_{x y}=$ $-\eta \partial u_{x} / \partial y$. Inserting the resulting distribution function in (21) and carrying out the integration over angles we can perform the energy integration analytically, using the fact that

$$
\frac{\xi^{2}}{E^{2}}=1-\frac{\Delta^{2}}{\xi^{2}+\Delta^{2}} \simeq 1-\pi \Delta \delta(\xi)
$$

Here the representation of the Lorentzian by a deltafunction is valid as long as the remaining part of the integrand varies slowly on the scale of $\Delta$, that is provided $\Delta \ll k T$. Since the value of $f^{0}\left(1-f^{0}\right)$ at the chemical potential is $1 / 4$ for a degenerate fermion gas we obtain

$$
\eta=\eta_{n}\left(1-\frac{\pi}{4} \frac{\Delta}{k T_{c}}\right)
$$

to lowest order in $\Delta / k T_{c}$. The quantity $\eta_{n}=m n v_{\mathrm{F}}^{2} \tau / 5$ is the viscosity in the normal state at $T_{c}$.

Secondly, the probability of two-quasiparticle scattering processes is modified by the coherence factors appearing in the Bogoliubov transformation. Since the number of quasiparticles is not a conserved quantity in the superfluid state, it is also necessary to include processes in which one quasiparticle decays into three as well as the inverse processes.

Taking both these effects into account, it is possible near the transition temperature to express the relative decrease in viscosity in terms of normal-state quantities and relate the coefficient in front of $\Delta / k T_{c}$ to the ratio between the viscous relaxation time $\tau_{\eta}$ and the lifetime $\tau(0)$ of a quasiparticle in the normal state at the Fermi energy. One finds [13] that the change in viscosity is given by

$$
\frac{\delta \eta}{\eta}=-\frac{\pi}{4}\left(1-\frac{\pi^{2}}{12}+\frac{\tau_{\eta}}{\tau(0)}\right)^{2} \frac{\tau(0)}{\tau_{\eta}} \frac{\Delta}{k T_{c}} .
$$

The coefficient of the $\Delta / k T_{c}$ term may thus be expressed solely in terms of normal-state quantities. For the trial function (3) used here, the ratio between the lifetime and the viscous relaxation time is [14]

$$
\frac{\tau(0)}{\tau_{\eta}}=2\left(\int_{0}^{1} d x \frac{x^{5}}{\sqrt{1-x^{2}}} \frac{d \sigma}{d \Omega}\right)\left(\int_{0}^{1} d x \frac{x}{\sqrt{1-x^{2}}} \frac{d \sigma}{d \Omega}\right)^{-1} .
$$

The medium cross section diverges at zero COM momentum at $T_{c}$. This leads to a non-integrable pole at $x=1$ in both integrals in (25) and therefore

$$
\frac{\tau(0)}{\tau_{\eta}}=2
$$

This is a universal result, independent of the coupling strength, coming from the Cooper instability at $T_{c}$.

The viscous relaxation time is obtained from the viscosity by removing the factor $(X, X)$, where $X$ is the trial function proportional to $v_{y} p_{x}$. By the same reasoning that led to (23) it follows that the value $(X, X)_{s}$ of the scalar product in the superfluid state is related to its value $(X, X)_{n}$ in the normal state by

$$
(X, X)_{s} \simeq(X, X)_{n}\left(1-\frac{\pi}{4} \frac{\Delta}{k T_{c}}\right)
$$

to leading order in $\Delta / k T_{c}$. Consequently, the change in $\tau_{\eta}$ is given by

$$
\frac{\delta \tau_{\eta}}{\tau_{\eta}}=0.0643 \frac{\Delta}{k T_{c}} .
$$

Due to the smallness of the numerical factor in (28) we conclude that there is very little change in the viscous relaxation rate upon entering the superfluid phase. 


\section{TRAPPED GAS}

We now present results for the thermal relaxation rate as a function of temperature both in the weak and the resonant coupling limit for a trapped two-component Fermi gas. To keep the number of trapped particles $N=2 N_{\sigma}$ constant (there are $N_{\sigma}$ atoms in each hyperfine state), we calculate $\Omega$ in the ladder approx. as in Sec. [IV and the chemical potential is determined by the condition $N=-\partial_{\mu} \Omega$. The relaxation rate is then calculated from (4). The presence of the trapping potential $V(\mathbf{r})$ is treated in the Thomas-Fermi approximation by a local chemical potential $\mu(\mathbf{r})=\mu-V(\mathbf{r})$. This requires an additional integration over the spatial coordinates $\mathbf{r}$, and the resulting multidimensional integrals are evaluated using a Monte-Carlo routine.

\section{A. Low and high temperature limits}

As for the homogeneous case, the integrals in (4) for the thermal relaxation rate can be calculated analytically in the low and high temperature limits using the unitarized vacuum form $\mathcal{T}_{\text {uni }}$ for the $\mathcal{T}$ matrix. For $T / T_{\mathrm{F}} \ll 1$ and weak coupling $k_{\mathrm{F}}|a| \ll 1$, we obtain

$$
\frac{1}{\tau_{T}}=\frac{16 \pi(k T)^{2}}{15 \hbar^{3}} m a^{2}
$$

For high temperatures, $T \gg T_{\mathrm{F}}$, we obtain

$$
\begin{gathered}
\frac{1}{\tau_{T}}=\frac{N}{10 \pi^{2}} \frac{m \tilde{\omega}^{3} \bar{\sigma}}{k T} \\
=\frac{2 N \tilde{\omega}^{3}}{5 \pi k T} \times \begin{cases}a^{2} & , T \ll T_{a} \\
\hbar^{2} /(3 m k T) & , T \gg T_{a}\end{cases}
\end{gathered}
$$

with $\tilde{\omega}^{3}=\omega_{x} \omega_{y} \omega_{z}$ and $\bar{\sigma}$ given by 15 . As for the homogeneous case, $\mathcal{T}=\mathcal{T}_{\text {uni }}$ is a good approximation at low temperatures for weak coupling only, whereas it is valid at high temperatures for all coupling strengths.

\section{B. Numerical results}

For weak coupling $k_{\mathrm{F}}|a| \ll 1$, the numerical calculations confirm as expected that there are no effects of the medium (except for very small $T$ close to $T_{c}$ ) and the results for the thermal relaxation rate are identical to the ones reported in Fig. 2 of $\mathrm{I}$.

We therefore concentrate on the strongly interacting limit. Fig. 3 shows the thermal relaxation rate $\tau_{T}^{-1}$ as a function of temperature for a strongly interacting system with $k_{\mathrm{F}}|a| \gg 1$. The rate is in units of $\epsilon_{\mathrm{F}} / \hbar$ where $\epsilon_{\mathrm{F}}=\hbar \tilde{\omega}(3 N)^{1 / 3}$ is the Fermi energy for $N$ noninteracting trapped particles. We have chosen parameters in (10) corresponding to a resonant interaction with $k_{\mathrm{F}} a=-11.8$ and a small effective range $k_{\mathrm{F}} r_{\text {eff }} \ll 1$. As for the homogeneous case, the strong interaction gives

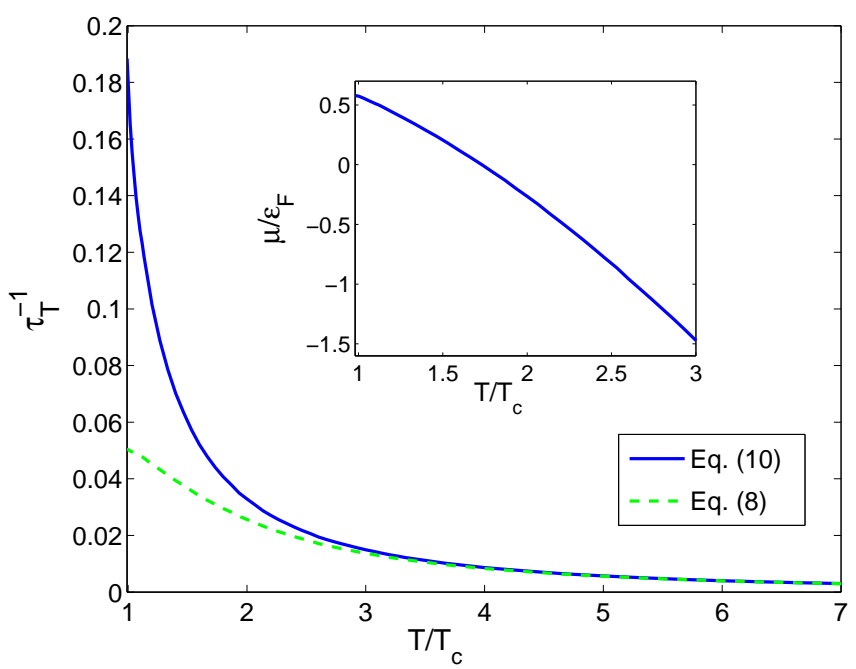

FIG. 3: (Color online) The thermal relaxation rate in a trap in units of $\epsilon_{\mathrm{F}} / \hbar$ for the resonant coupling case $k_{\mathrm{F}}|a| \gg 1$. The solid line is the numerical result with the full scattering matrix in (10), the dashed line the numerical result using the vacuum approximation (8) for the scattering matrix. The curves are given for $T \geq T_{c} \approx 0.3 T_{\mathrm{F}}$.

rise to a superfluid transition below a critical temperature $T_{c}$. We find $T_{c} \approx 0.3 T_{\mathrm{F}}$ at resonance $\left(k_{\mathrm{F}}|a| \gg 1\right)$ in excellent agreement with the results in Ref. [15].

We see from Fig. 3 by comparing the solid and the dashed lines, that the medium increases the relaxation rate significantly for low temperatures as compared to the vacuum prediction: close to $T_{c}$ the rate is approximately 3.6 times higher than the vacuum result. The increase is smaller than for the homogeneous case since the effects of the medium are reduced near the edge of the cloud where the density is reduced. The reason for the significant increase of the rate is of course identical to the effect discussed for the homogeneous case: the medium increase of the resonant scattering rate for low temperatures $\left(T-T_{c}\right) / T_{c} \lesssim 1$ signals the Cooper instability at $T_{c}$. For high temperatures, the medium effects are negligible. As for the homogeneous case, the $T^{2}$ dependence of the rate coming from the Fermi blocking of the available scattering states for $T / T_{\mathrm{F}} \ll 1$ is not observed for $T \geq T_{c}$ since $k T_{c} / \mu\left(T_{c}\right) \approx 0.5$ is rather high.

Fig. [3] shows the maximum relaxation rate for a twocomponent trapped Fermi gas for $k_{\mathrm{F}}|a| \rightarrow \infty$. The large difference between the relaxation rate for a weakly interacting system and a resonantly interacting system should be contrasted with the much smaller effects of the resonant interaction on thermodynamic properties.

\section{Experiments}

Let us comment on the possible experimental observation of the effects described above. The thermal relaxation rate can be measured by "heating" the gas pref- 
erentially in one spatial direction followed by observing the time evolution towards equilibrium with a uniform temperature in all directions 1, 2]. These experiments have already demonstrated the saturation of the scattering rate at resonance in the classical regime. By performing such experiments for decreasing temperatures, one should observe a significant increase in the rethermalization rate as compared to the vacuum rate when $\left(T-T_{c}\right) / T_{c} \lesssim 1$.

\section{CONCLUSION}

Using a variational approach, we have calculated the viscosity and the thermal relaxation rate of an interact- ing Fermi gas for weak and for strong coupling. Both homogeneous and trapped systems were considered and medium effects on the scattering properties were taken into account. The rates were shown to be significantly increased for temperatures close to the superfluid transition temperature. This is due to the presence of the medium and signals the onset of Cooper pairing at $T_{c}$. We furthermore considered the effects of superfluidity below $T_{c}$ to leading order in the energy gap. The effects described in this paper can be measured directly by rethermalization experiments.
[1] T. Loftus, C. A. Regal, C. Ticknor, J. L. Bohn, and D. S. Jin, Phys. Rev. Lett. 88, 173201 (2002).

[2] C. A. Regal, C. Ticknor, J. L. Bohn, and D. S. Jin, Phys. Rev. Lett. 90, 053201 (2003).

[3] P. Massignan, G. M. Bruun, and H. Smith, Phys. Rev. A 71, 033607 (2005).

[4] C. J. Pethick and H. Smith, Bose-Einstein Condensation in Dilute Gases (Cambridge University Press, Cambridge 2002), p. 310.

[5] G. M. Bruun, A. D. Jackson, and E. E. Kolomeitsev, cond-mat/0501407 to appear in Phys. Rev. A.

[6] G. M. Bruun, Phys. Rev. A 70, 053602 (2004).

[7] G. M. Bruun and C. J. Pethick, Phys. Rev. Lett. 92, 140404 (2004).

[8] See e.g. G. Baym and C. J. Pethick, Landau Fermi-Liquid
Theory (John Wiley, 1991) Sec. 1.2.4.

[9] J. N. Milstein, S. J. J. M. F. Kokkelmans, and M. J. Holland, Phys. Rev. A 66, 043604 (2002).

[10] P. Pieri, L. Pisani, and G. C. Strinati, cond-mat/0410578

[11] A. Peshier and W. Cassing, hep-ph/0502138

[12] D. Vollhardt and P. Wölfle, The Superfluid Phases of Helium 3, (Taylor and Francis, London, 1990).

[13] P. Bhattacharyya, C. J. Pethick, and H. Smith, Phys. Rev. B 15, 3367 (1977).

[14] H. Smith and H. H. Jensen, Transport Phenomena (Oxford University Press, Oxford 1989).

[15] A. Perali, P. Pieri, L. Pisani, and G. C. Strinati, Phys. Rev. Lett. 92, 220404 (2004). 